\title{
Genetic relatedness of selected clinical Vibrio cholerae 0139 isolates from the southern coastal area of China over a 20-year period
}

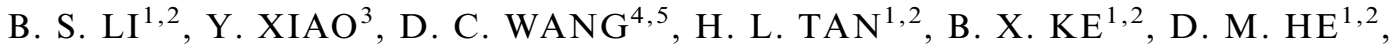 \\ C. W. KE ${ }^{1,2}$ AND Y. H. ZHANG ${ }^{1,2 *}$ \\ ${ }^{1}$ Key Laboratory for Repository and Application of Pathogenic Microbiology, Research Center for Pathogens \\ Detection Technology of Emerging Infectious Diseases, Guangdong Provincial Center for Disease Control and \\ Prevention, Guangzhou, China \\ ${ }^{2}$ WHO Collaborating Centre for Surveillance, Research and Training of Emerging Infectious Diseases \\ ${ }^{3}$ School of Public Health and Tropical Medicine, Southern Medical University, Guangzhou, China \\ ${ }^{4}$ State Key Laboratory of Infectious Disease Prevention and Control, National Institute for Communicable \\ Disease Control and Prevention, China CDC, Beijing, China \\ ${ }^{5}$ Collaborative Innovation Center for Diagnosis and Treatment of Infectious Diseases, Hangzhou, China
}

Received 14 September 2015; Final revision 28 April 2016; Accepted 3 May 2016;

first published online 16 June 2016

\section{SUMMARY}

Vibrio cholerae O139 emerged as a causative agent of epidemic cholera in 1992 in India and Bangladesh, and was subsequently reported in China in 1993. The genetic relatedness and molecular characteristics of $V$. cholerae O139 in Guangdong Province, located in the southern coastal area of China, remains undetermined. In this study, we investigated 136 clinical $V$. cholerae O139 isolates from 1993 to 2013 in Guangdong. By conventional PCR, 123 (90.4\%) isolates were positive for $c t x B$, ace and zot. Sequencing of the positive amplicons indicated 113 $(91 \cdot 7 \%)$ isolates possessed the El Tor allele of $\operatorname{ct} x B$ (genotype 3); seven carried the classical $\operatorname{ct} x B$ type (genotype 1) and three harboured a novel $\operatorname{ctx} B$ type (genotype 5). With respect to $\operatorname{tcp} A, 123$ $(90 \cdot 4 \%)$ isolates were positive for the El Tor allele. In addition, pulsed-field gel electrophoresis (with NotI digestion) differentiated the isolates into clusters A and B. Cluster A contained seven of the non-toxigenic isolates from 1998 to 2000; another six non-toxigenic isolates (from 1998 and 2007) and all of the toxigenic isolates formed cluster B. Our results suggest that over a 20year period, the predominant $\mathrm{O} 139$ clinical isolates have maintained a relatively tight clonal structure, although some genetic variance and shift has occurred. Our data highlight the persistence of toxigenic $V$. cholerae $\mathrm{O} 139$ in clinical settings in the southern coastal area of China.

Key words: Bacterial typing, clinical microbiology, epidemics, Vibrio cholerae.

\section{INTRODUCTION}

Vibrio cholerae $\mathrm{O} 139$ Bengal was recognized as a second $V$. cholerae serotype capable of causing epidemic

\footnotetext{
* Author for correspondence: Dr Y. H. Zhang, 160 Qunxian Road, Dashi Street, Panyu District, Guangzhou, Guangdong 511430, China.

(Email: zyh@cdcp.org.cn)
}

cholera in 1992, after emerging in India, and rapidly spreading across multiple Asian countries [1, 2]. Genetic characterization of $V$. cholerae $\mathrm{O} 139$ isolates revealed that they were identical to $V$. cholerae $\mathrm{O} 1$ El Tor isolates, except for the substitution of a $35-\mathrm{kb}$ region of DNA encoding the O139 surface polysaccharide for the 22-kb $r f b$ region in $\mathrm{O} 1$ isolates [3]. Thus, V. cholerae O139 carried the same virulence 
factors found in O1, including the CTX prophage, toxin co-regulated pilus A (tcpA) and Vibrio seventh pandemic island I (VSP-I) and VSP-II [4].

$V$. cholerae $\mathrm{O} 1$ includes the Classical and $\mathrm{El}$ Tor biotype. Classical biotype strain was responsible for first six pandemics of cholera, whereas the other biotype was responsible for the current seventh pandemic. In addition to phenotypic traits, alleles of the cholera toxin subunit B $(\operatorname{ct} x B)$ of CTX prophage differ among these biotypes. Nine $\operatorname{ct} x B$ genotypes have been identified in the $\mathrm{O} 1 \mathrm{~V}$. cholerae isolates based on amino-acid residue substitutions [5]. Genotype 3 is associated with $V$. cholerae $\mathrm{O} 1 \mathrm{El}$ Tor isolates from the seventh pandemic, genotype 2 has only been found in El Tor isolates from Australia, and recently there has been recognition of El Tor biotype strains that carry an atypical variant of the CTX phage harbouring the classical $\operatorname{ct} x B$ (genotype 1) [6]. Other variants of $\operatorname{ctx} B$ have been reported in association with $\mathrm{O} 139$ strains in Bangladesh [7] and El Tor O1 strains in China [8]. Interestingly, although $\mathrm{O} 139$ isolates still co-exist with the $\mathrm{O} 1 \mathrm{El}$ Tor isolates in China, there is no evidence of El Tor $\operatorname{ctx} B$ variant genotypes in $\mathrm{O} 139$ isolates, which remain solely as genotype 3.

Located in the southern coastal area of China on the same latitude as Bengal, India, Guangdong Province is recognized as an historical origin of epidemic cholera. In July 1961, the first outbreak caused by $V$. cholerae $\mathrm{O} 1 \mathrm{El}$ Tor documented in China occurred in Yangjiang city, Guangdong, triggering the beginning of the seventh cholera pandemic in China [9]. The first report of $V$. cholerae O139 isolated in China was from May 1993, in Xinjiang Province [10], followed by outbreaks in multiple provinces in China over subsequent years. In China, V. cholerae O139 cases have been sporadic and have not resulted in epidemic or pandemic spread. V. cholerae O139 isolates continue to persist in Guangdong, although $V$. cholerae $\mathrm{El}$ Tor $\mathrm{O} 1$ isolates remain dominant. [11].

$V$. cholerae $\mathrm{O} 139$ has largely disappeared from countries where it emerged except for China and Vietnam. It is unclear what has caused the survival of O139 isolates in these regions. To identify the mechanisms and factors behind the persistence, we conducted molecular characterization of $V$. cholerae O139 isolates collected from Guangdong Province over the past 20 years. In order to determine their genotypes and depict genetic relatedness between isolates, a total of $136 \mathrm{~V}$. cholerae $\mathrm{O} 139$ isolates collected from 1993 to 2013 were characterized by polymerase chain reaction (PCR), gene sequencing of virulenceassociated genes and pulsed-field gel electrophoresis (PFGE).

\section{MATERIALS AND METHODS}

\section{Bacterial strains}

A total of $136 \mathrm{~V}$. cholerae $\mathrm{O} 139$ isolates collected from local laboratories were included in this study. Isolates were selected from each year and each city where cholera occurred in Guangdong Province from 1993 to 2013 (Table 1). All isolates used in this study had not been reported in previous studies. The isolates were identified as $V$. cholerae using conventional bacteriological methods [12]. $V$. cholerae O139 (ATCC 51394, MO45) was used as a reference strain in the study. All isolates were examined using the oxidase test, string test and triple sugar iron agar (TSI) reaction [12]. Serotyping was determined by slide agglutination with an $\mathrm{O} 139$ antiserum according to manufacturer's instructions (Denka Seiken, Japan).

\section{Chromosomal DNA preparation}

DNA was extracted from bacterial isolates using the QIAamp DNA Mini kit according to the manufacturer's instructions (Qiagen Inc., China). DNA extracts were dissolved in TE buffer $[10 \mathrm{~mm}$ Tris$\mathrm{HCl}, 0 \cdot 10 \mathrm{~mm}$ EDTA (pH 8.0)] and stored at $4{ }^{\circ} \mathrm{C}$ until required. Dilutions of template DNA were made with double sterile distilled water to obtain a final concentration of $\sim 100 \mathrm{ng} / \mathrm{ml}$.

\section{PCR and sequencing analysis}

PCR assays were performed using conventional PCR amplification. Target genes included cholera toxin B subunit $(\operatorname{ct} x B)$, accessory cholera enterotoxin (ace), and zonula occludens toxin (zot) of the CTX prophage [13], and the classical and El Tor-specific tcpA and tcpI genes of the toxin-coregulated pilus (TCP) pathogenicity island [14]. Additional putative accessory virulence genes included haemolysin $(h l y A)$ [15], outer membrane protein (ompU) [15], RTX toxin $(r t x C)$ gene [16] and heat-stable enterotoxin (st) [13]. Table 2 shows the primer sequences and their origins. PCR was performed with a thermal cycler T-100 (BioRad Laboratories, USA). V. cholerae O139 strain MO45 (ATCC 51394) was used as the positive control of PCR. Amplified products were separated on a $1 \%$ 
Table 1. The distribution and information of strains and $\operatorname{ctx} B$, tcpA genotypes in this study

\begin{tabular}{|c|c|c|c|c|}
\hline \multirow[b]{2}{*}{ Year } & \multirow[b]{2}{*}{ No. of isolates } & \multirow[b]{2}{*}{ County (no. of isolates) } & \multicolumn{2}{|l|}{ Virulence genes genotype (G) } \\
\hline & & & $\operatorname{ctx} B$ & tcp $A$ \\
\hline 1993 & 2 & FS (1), SZ (1) & G3 & ET \\
\hline 1994 & 8 & GZ (5), ZJ (1), HZ (1), FS (1) & G3, G1 [GZ (4)] & ET \\
\hline 1995 & 6 & GZ (5), FS (1) & G3 & ET \\
\hline 1996 & 7 & GZ (6), ZH (1) & G3 & ET \\
\hline 1997 & 20 & GZ (20) & G3, G1 [GZ (1)] & ET \\
\hline 1998 & 13 & GZ (6), FS (4), SZ (2), HZ (1) & G3 & ET \\
\hline 1999 & 30 & GZ (11), FS (9), SZ (4), ZS(3), JM(2), DG(1) & G3, G1 [DG (1), GZ (1)], G5 [GZ (3)] & ET \\
\hline 2000 & 3 & GZ (1), SZ (1), JM (1) & G3 & ET \\
\hline 2001 & 11 & FS (6), GZ (2), ZJ (2), SZ (1) & G3 & ET \\
\hline 2002 & 2 & GZ (2) & G3 & ET \\
\hline 2003 & 11 & GZ (11) & G3 & ET \\
\hline 2004 & 3 & GZ (1), ZJ (1), ZS (1) & G3 & ET \\
\hline 2005 & 5 & GZ (1), ZJ (1), FS (1), YJ (1), MM (1) & G3 & ET \\
\hline 2006 & 3 & MM (2), ZS (1) & G3 & ET \\
\hline 2007 & 3 & GZ (1), ZJ (1), SZ (1) & G3 & ET \\
\hline 2008 & 3 & DG (2), SZ (1) & G3 & ET \\
\hline 2010 & 1 & GZ (1) & G3 & ET \\
\hline 2012 & 3 & GZ (1), MM (1), FS (1) & G3 & ET \\
\hline 2013 & 2 & $\mathrm{ZJ}(1), \mathrm{MM}(1)$ & G3 & ET \\
\hline Total & 136 & & & \\
\hline
\end{tabular}

DG, Dongguang county; ET, El Tor biotype; FS, Foshan county; GZ, Guangzhou county; HZ, Huzhou county; JM, Jiangmen county; MM, Maoming county; SZ, Shenzhen county; YJ, Yangjiang county; ZH, Zhuhai county; ZJ, Zhanjiang county; ZS, Zhongshan county.

agarose gel, stained with $1 \%$ ethidium bromide, and image captured by Gel Doc EQ (Bio-Rad Laboratories). Amplicons were sequenced by a commercial supplier (Shenggong Inc., China). Sequences were compared using BioEdit software v. 7.0 (Ibis Biosciences, USA). Clustal-W was used to perform multiple nucleotide alignments. A phylogenetic tree was conducted by MEGA 5.0 software using unweighted pair-group method with arithmetic mean (UPGMA), bootstrap values were calculated based on 1000 replicates. The reference sequences of different types of $c t x B$ were obtained from GenBank (accession nos. KC754362-KC754371).

\section{PFGE analysis}

PFGE was performed according to the PulseNet standardized PFGE protocol [17]. Briefly, the enzyme digestion for each plug used $40 \mathrm{U}$ of $\operatorname{Not} \mathrm{I}$ at $37^{\circ} \mathrm{C}$ for $4 \mathrm{~h}$ (Promega Molecular Biochemicals, USA). Salmonella enterica serotype Braenderup H9812 was used as a DNA molecular weight standard. Restriction fragments were separated using a CHEF Mapper (Bio-Rad Laboratories). Running times consisted of block I ( $13 \mathrm{~h}$ and a linear ramp of 2-10 s) and block II ( $6 \mathrm{~h}$ and a linear ramp of $20-25 \mathrm{~s}$ ), both blocks used a voltage gradient of $6 \mathrm{~V} / \mathrm{cm}$. Gels were stained with $1 \mathrm{mg} / \mathrm{ml}$ of ethidium bromide solution (Sigma, USA) and destained with reagent grade water. Images were captured with a Gel Doc EQ (Bio-Rad Laboratories). PFGE patterns were analysed using BioNumerics software v. 6.6 (Applied Maths BVBA, Belgium). Dendrograms were generated using the Dice coefficient and UPGMA.

\section{RESULTS}

\section{Distribution of of $c t x B$ genotypes}

Of the 136 tested O139 isolates, $123(90 \cdot 4 \%)$ harboured $c t x B$; the phylogenetic tree of 123 ctxB sequences revealing multiple genotypes (Fig. 1). The majority of isolates $(n=113,91.7 \%)$ belonged to $\operatorname{ctx} B$ genotype 3 (El Tor type), seven isolates (LETV3186, 3187, 3188, 3190, 3433, 3644, 3766) carried genotype 1 (Classical type; four from 1994, one from 1997, two from 1999), and three isolates (L-ETV3655, 3658, 3659) from 1999 carried genotype 5. 
Table 2. Primers used in this study for detection of virulence-associated genes

\begin{tabular}{|c|c|c|c|}
\hline Target & Nucleotide sequence $\left(5^{\prime}-3^{\prime}\right)$ & Amplicon size (bp) & Ref. \\
\hline $\operatorname{ctx} B-\mathrm{F}$ & GCCGGGTTGTGGGAATGCTCCAAG & 536 & [13] \\
\hline $\operatorname{ctx} B-\mathrm{R}$ & CATGCGATTGCCGCAATTAGTATGGC & & \\
\hline ace- $\mathrm{F}$ & TAAGGATGTGCTTATGATGGACACCC & 316 & [13] \\
\hline ace- $\mathrm{R}$ & CGTGATGAATAAAGATACTCATAGG & & \\
\hline$z o t-\mathrm{F}$ & TCGCTTAACGATGGCGCGTTTT & 947 & [13] \\
\hline$z o t-\mathrm{R}$ & AACCCCGTTTCACTTCTACCCA & & \\
\hline$s t-\mathrm{F}$ & GAGAAACCTATTCATTGC & 216 & [13] \\
\hline$s t-\mathrm{R}$ & GCAAGCTGGATTGCAAC & & \\
\hline tсp $A-\mathrm{F}$ & CACGATAAGAAAACCGGTCAAGAG & 453 & [14] \\
\hline tсpA-R & CGAAAGCACCTTCTTTCACACGTTG & & \\
\hline tcp $I-\mathrm{F}$ & TAGCCTTAGTTCTCAGCAGGCA & 862 & [14] \\
\hline tcpI-R & GGCAATAGTGTCGAGCTCGTTA & & \\
\hline omp $U-\mathrm{F}$ & ACGCTGACGGAATCAACCAAAG & 869 & [15] \\
\hline omp $U-\mathrm{R}$ & GCGGAAGTTTGGCTTGAAGTAG & & \\
\hline hlyA-F & GAGCCGGCATTCATCTGAAT & 481 & [15] \\
\hline hly $A-\mathrm{R}$ & CTCAGCGGGCTAATACGGTTTA & & \\
\hline $\operatorname{rtx} C-\mathrm{F}$ & CGACGAAGATCATTGACGAC & 265 & [16] \\
\hline $\operatorname{rtx} C-\mathrm{R}$ & CATCGTCGTTATGTGGTTGC & & \\
\hline
\end{tabular}

F, Forward; R, reverse.

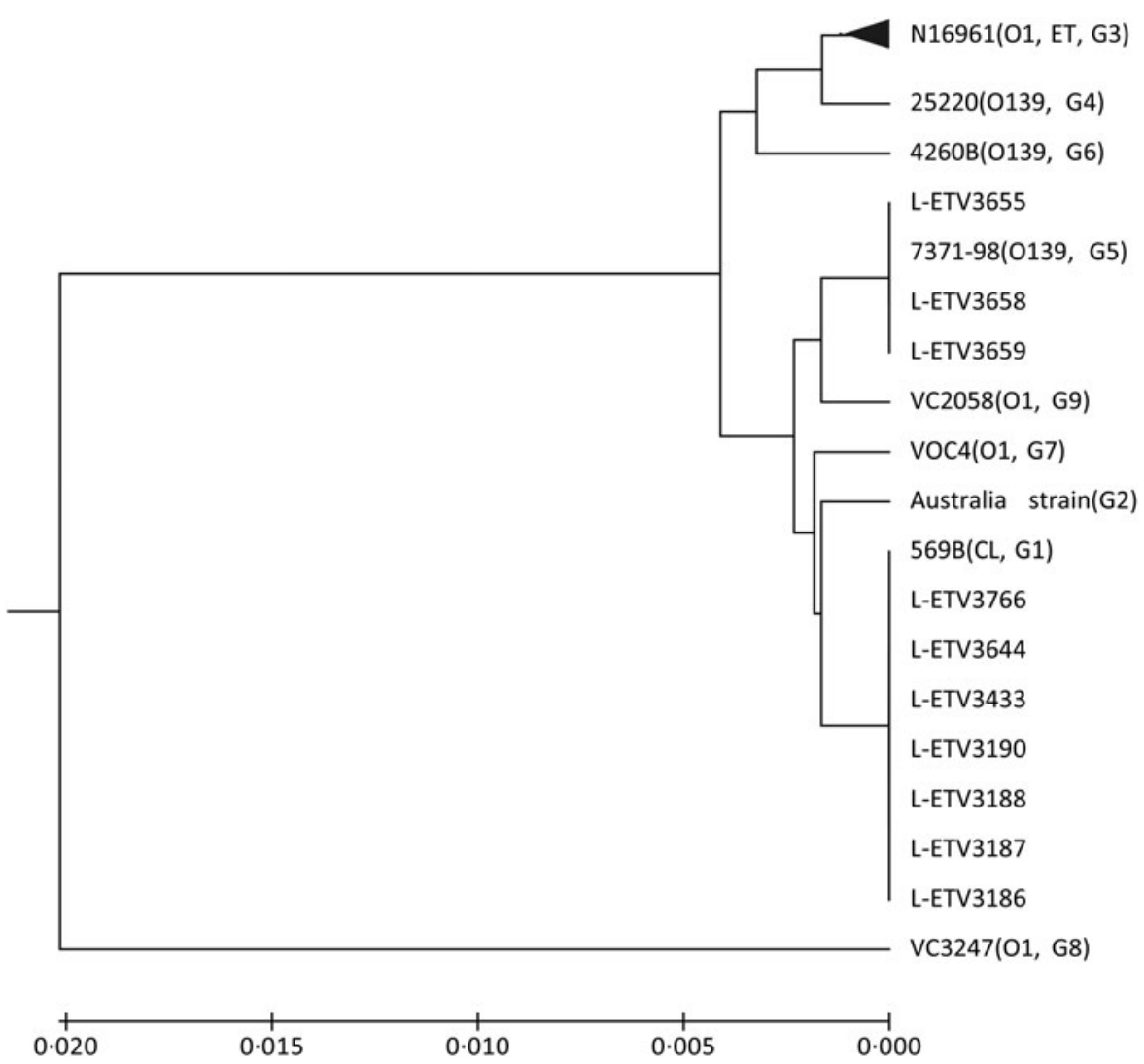

Fig. 1. Phylogenetic tree of $c t x B$ genotypes of this study. The tree was constructed by unweighted pair-group method with arithmetic mean based on $c t x B$ amino-acid sequences. One thousand bootstrap replicates were performed for each analysis. The reference amino-acid sequences of different $c t x B$ were from GenBank. G1-G9, genotypes 1-9. The black triangle represents 113 isolates belonging to $\operatorname{ctx} B$ genotype 3 . 


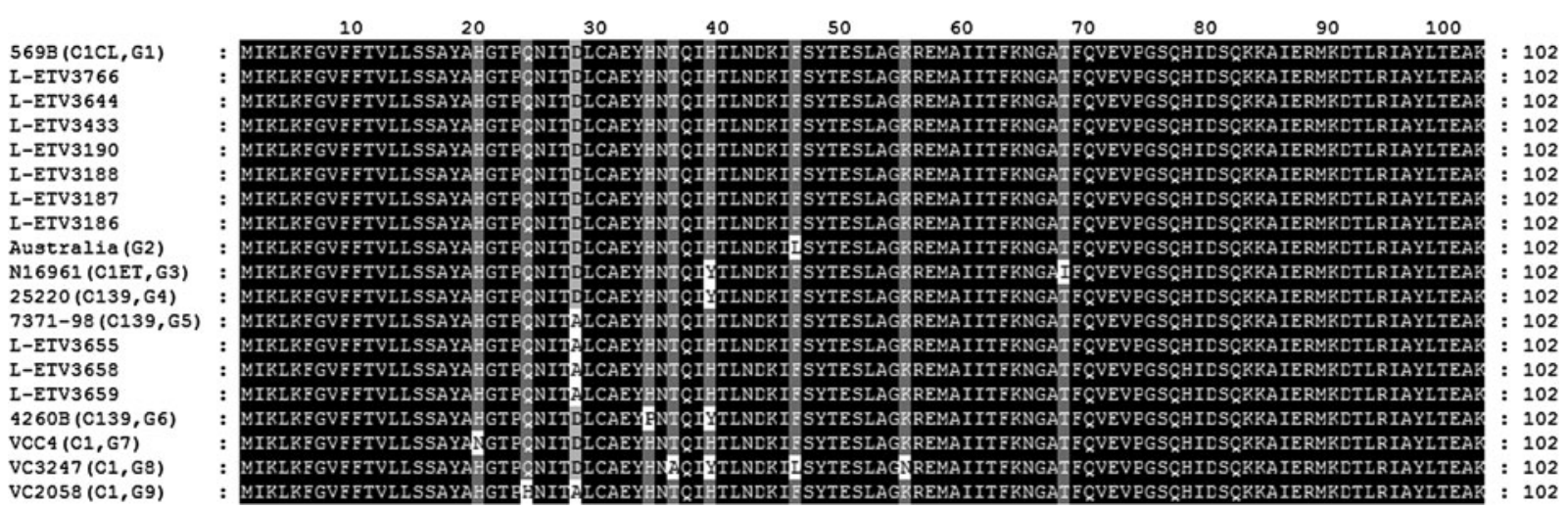

Fig. 2. Amino-acid sequence alignments of $\operatorname{ctx} B$. The reference sequences for $\operatorname{ct} x B$ are shown within parentheses including their serogroup and genotypes. Sequence characters in black on a white background represent rare substitutions; white on a grey background represent common substitutions; white on a black background represent identical sequences in all genotypes. ET, El Tor biotype; CL, Classical biotype. G1-G9, genotypes 1-9.

Based on putative amino-acid sequence of the $\operatorname{ctx} B$ subunit, all genotype 3 isolates in this study were identified as similar to reference strain N16961 (El Tor biotype), which results in the following amino-acid sequence: $\mathrm{His}^{20}-\mathrm{Gln}^{24}-\mathrm{Asp}^{28}-\mathrm{His}^{34}-\mathrm{Tyr}^{39}-\mathrm{Phe}^{46}-\mathrm{Lys}^{55}$ $\mathrm{Ile}^{68}$ (Fig. 2). Seven genotype 1 and three genotype 5 isolates were similar to genotype 3 , respectively, with the exception of two loci for $\mathrm{His}^{39}-\mathrm{Thr}^{68}$ and three loci for $\mathrm{Ala}^{28}-\mathrm{His}^{39}-\mathrm{Thr}^{68}$.

\section{Identification of virulence-associated genes}

The $123 \operatorname{ctx} B$-positive isolates were also positive for ace and zot; another two ORFs within the CTX prophage. Isolates negative for $\operatorname{ct} x B$ were also negative for the two phage accessory genes (Table 3). Similarly, 123 isolates yielded PCR-positive results for $t c p A$, and all amplicons were subsequently sequenced and identified as El Tor type of tcpA. Twenty-eight tcp $A$-positive isolates were positive for tcpI; another ORF within the TCP pathogenicity island. PCR-based analyses revealed unanticipated variations in the distribution of putative accessory virulence genes, including $r t x C(97 \cdot 8 \%)$, hlyA (96.3\%) and ompU $(75.0 \%)$. The three $r t x C$-negative isolates included two strains from 2004 and one strain from a patient identified in 2012. In addition, all the isolates were $s t$-negative.

\section{PFGE patterns}

All 136 isolates were differentiated into 75 distinct patterns by $\operatorname{Not} \mathrm{I}$ endonuclease digestion (Fig. 3).
Table 3. Characteristics of CTX prophage/TCP genes and relationship with putative accessory virulence genes

\begin{tabular}{|c|c|c|c|c|c|c|c|c|c|}
\hline \multirow{2}{*}{$\begin{array}{l}\text { No. of } \\
\text { strain(s) }\end{array}$} & \multicolumn{3}{|c|}{$\begin{array}{l}\text { CTX } \\
\text { prophage }\end{array}$} & \multicolumn{2}{|l|}{ TCP } & \multicolumn{4}{|c|}{$\begin{array}{l}\text { Putative accessory } \\
\text { virulence genes }\end{array}$} \\
\hline & $\operatorname{ctx} B$ & ace & zot & tcp $A$ & $t c p I$ & $\operatorname{rtx} C$ & ompU & hly $A$ & $s t$ \\
\hline 71 & + & + & + & + & - & + & + & + & - \\
\hline 27 & + & + & + & + & + & + & + & + & - \\
\hline 14 & + & + & + & + & - & + & - & + & - \\
\hline 4 & + & + & + & + & - & + & - & + & - \\
\hline 2 & + & + & + & + & - & - & + & + & - \\
\hline 3 & + & + & + & + & - & + & - & - & - \\
\hline 1 & + & + & + & + & - & + & + & - & - \\
\hline 1 & + & + & + & + & + & + & - & + & - \\
\hline 10 & - & - & - & - & - & + & - & + & - \\
\hline 1 & - & - & - & - & - & + & - & - & - \\
\hline 1 & - & - & - & - & - & - & - & + & - \\
\hline 1 & - & - & - & - & - & + & + & + & - \\
\hline
\end{tabular}

+ , Positive; - , negative.

Phylogenetic analysis using UPGMA clustered the patterns into two clusters: A and B. Cluster A contained seven of the $\mathrm{O} 139$ non-toxigenic isolates from 1998 to 2000 and each of the seven non-toxigenic isolates revealed a unique pattern. Cluster B contained the other group of non-toxigenic isolates (from 1998 and 2007) and all toxigenic isolates; 68 unique patterns were represented with an overall similarity of $82 \cdot 4 \%$.

All non-toxigenic isolates displayed one unique PFGE pattern, which demonstrated less overall similarity compared to toxigenic isolates. Within cluster $\mathrm{B}$, indistinguishable patterns were represented in 


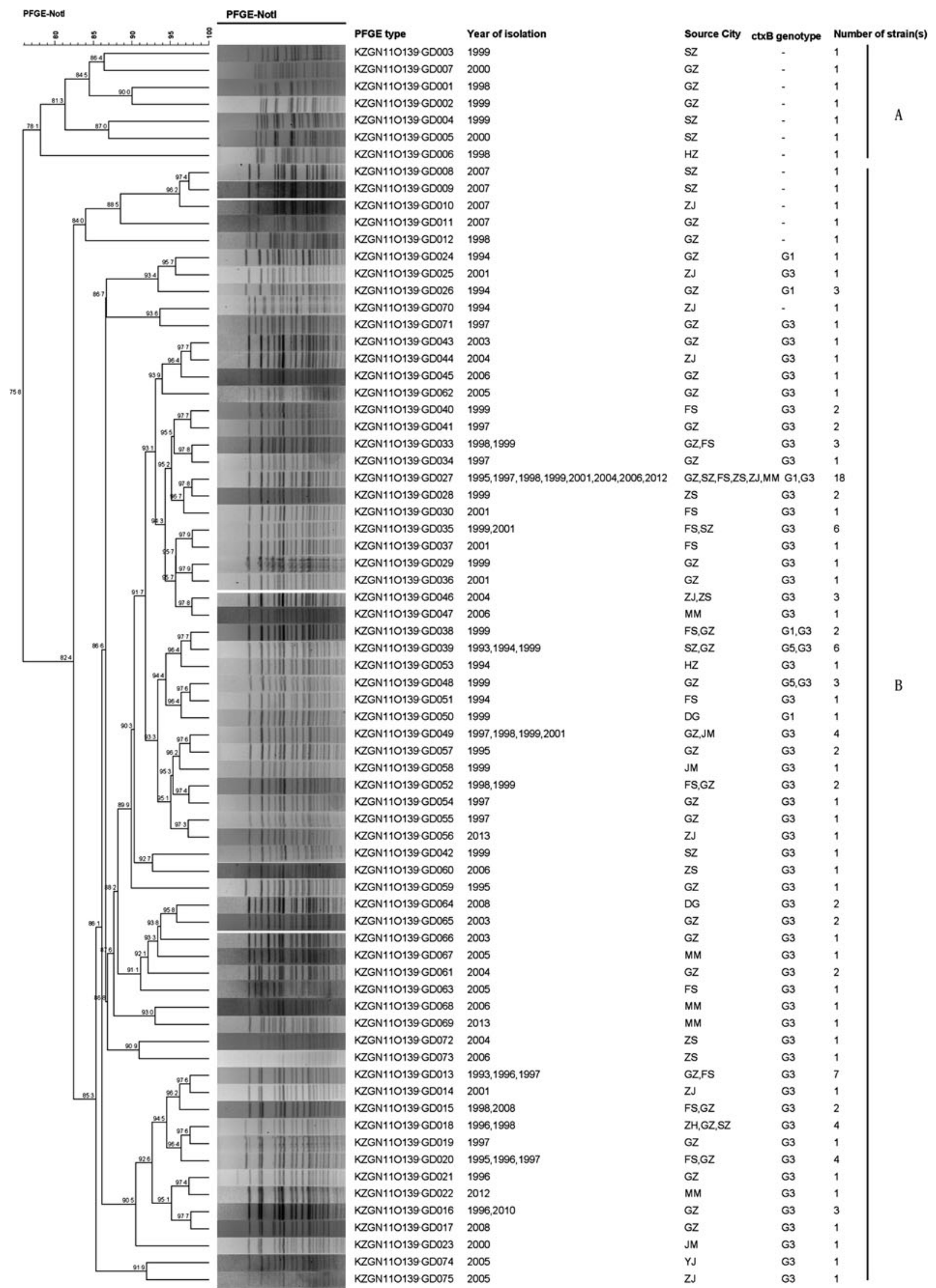

Fig. 3. For legend see next page. 
multiple years and from different regions. For example, among toxigenic isolates, the most common pattern was GD027, containing 18 isolates recovered in 1995-2012 from six cities. Moreover, isolates with the same PFGE types, GD013 (7), GD035 (6), GD039 (6), GD049 (4), GD018 (4) and GD020 (3), also appeared in different years and multiple cities. At the same time, multiple patterns were found in the same cities at the same time: GD019, GD034, GD041, GD054, GD055 and GD071 appeared in Guangzhou in 1997; and GD030 and GD037 appeared in Foshan in 2001. The first O139 isolates appeared in Foshan and Shenzhen in 1993 and displayed different PFGE patterns, suggesting different introductions, while the isolates from Shenzhen in 1993 shared the same PFGE pattern (GD039) with isolates from Guangzhou in 1994 and 1999, suggesting transmission.

Three $\operatorname{ctx} B$ genotype 5 isolates from 1999 in Guangzhou exhibited two PFGE patterns, GD039 and GD048. The GD039 pattern represented at least two cases and GD048 contained one case; these isolates shared $91.7 \%$ similarity. In contrast, the seven $\operatorname{ct} x B$ genotype 1 isolates were dispersed into patterns GD026, GD027, GD038, GD050 and three in GD024. These cases were identified in Guangzhou (one from 1997, one from 1999 and four from 1994) and Dongguan (one from 1999) in different years. All seven $\operatorname{ct} x B$ genotype 1 isolates shared $91.7 \%$ similarity. Two cases contained in pattern GD024 were considered epidemiological close contacts, suggesting a localized outbreak.

\section{DISCUSSION}

Until now, the variant types of $\operatorname{ctx} B$ were mainly found in $V$. cholerae $\mathrm{O} 1$ isolates, whereas only few variant genotypes were found in the O139 serogroup in Bangladesh [7]. Our previous study showed that the O139 isolates collected in China were entirely El Tor type (genotype 3 ) of $c t x B$ [11]. In this study, we detected genotype 1 (Classical type) and genotype 5 type of $\operatorname{ctx} B$ carried isolates from Guangdong Province, which to the best of our knowledge is the first report of variant types (but not El Tor type) of ctx $B$ from $V$. cholerae $\mathrm{O} 139$ isolates in China. Our study also indicated that the $\operatorname{ct} x B$ variants are not limited to the $\mathrm{O} 1$ serogroup in China. Furthermore, in this study the earliest variant $\operatorname{ctx} B$-type isolate emerged in 1994, which was even earlier than that from Bangladesh O139 isolates (in 1998) [7]. Thus, factors that induce changes in the $c t x B$ gene are unclear. From this study, the $\operatorname{ct} x B$ variant isolates were sporadically distributed in different periods and regions. It is important to investigate the molecular characteristics of $V$. cholerae $\mathrm{O} 139$ to identify the possible epidemic caused by this $c t x B$ variant isolate. A recent study reported that in Bangladesh and Haiti, the $\mathrm{O} 1 \mathrm{El}$ Tor clinical variant isolates produced increased cholera toxin and expressed higher virulence factors than El Tor wild-type isolates [18]. Meanwhile, the $\mathrm{O} 1 \mathrm{El}$ Tor isolates containing the classical $\operatorname{ctx} B$ allele have replaced the prototype El Tor in China in recent years [9], as well as in other Asian countries $[6,19,20]$. Therefore, it is necessary to strength the surveillance of the O139 serogroup, which is experiencing this potential genetic shifting. A previous study indicated that toxigenic (cholera toxin produced) O139 isolates maintained a relatively high clonality, although isolates collected from widely different geographical locations were non-toxigenic isolates; however, the non-toxigenic isolates exhibited greater diversity and multiple clonal lineages [11]. This feature was also identified by PFGE of our local isolates in this study. The predominant pattern of the toxigenic isolates was GD027, which contained 18 isolates from 1994 to 2012. However, the first documented O139 isolates in Guangdong were recovered from diarrhoeal cases in Shenzhen and Foshan in 1993. The PFGE pattern for the Shenzhen isolate was indistinguishable from two isolates recovered from Guangzhou in 1994 and 1999, while the isolate from Foshan was indistinguishable from one isolate recovered from Guangzhou in 1997. These data suggest that O139 isolates appear to survive and spread throughout the environment since their introduction in Guangdong in 1993.

Epidemiological data showed that three cases with $\operatorname{ct} x B$ genotype 5 (two PFGE GD039 and one GD048) were all sporadic and isolated from the

Fig. 3. Dendrogram constructed from the PFGE profiles generated from NotI-digested genomic DNA of $V$. cholerae O139 isolates in Guangdong Province, southern coastal area of China. The dendrogram was constructed by BioNumerics software v. 6.6 using the Dice coefficient and unweighted pair-group method with arithmetic mean algorithm (UPGMA). The scale shows percent similarity. 
same suburban village in Guangzhou in 1999. However, the seven $\operatorname{ct} x B$ genotype 1 isolates were dispersed into patterns GD026, GD027, GD038, GD050 (one case each) and three cases of pattern GD024, most of these cases presented in different years and geographical locations. Although PFGE is the 'gold standard' for identification and traceability of many outbreaks, PFGE patterns produced by restriction enzyme digestion only described a small part of the genome variation with limited resolution, especially for high clonality groups.

$V$. cholerae $\mathrm{O} 139$ first emerged in India and Bangladesh in 1992, resulting in a new subwave of cholera in Southeast Asia, including China. The surveillance data presented here represent sporadic cases of cholera caused by $\mathrm{O} 139$ in Guangdong and were not associated with any large outbreaks or epidemics over a 20-year time period. Epidemiologically only one outbreak associated with O139 occurred in the region (Foshan city, 2001), which included 15 cases from a dinner party. Outbreak isolates shared a common PFGE pattern (GD035); this pattern was observed as early as 1999 in Foshan and Shenzhen. Additional possible small outbreaks, based on identification of some cases with a clear epidemiological links and displaying the same PFGE patterns, were also identified. Overall, this study, together with other reports in China [21-23], indicated that $V$. cholerae O139 in China appears to be associated with local outbreaks, particularly with foodborne outbreaks in various regions. Meanwhile, the predominant O139 clinical isolates have maintained a relatively tight clonal structure, although some genetic variance and shift has occurred. This maybe lead to the continual contamination and existence of toxigenic O139 in environment and seafood, suggesting the risk of cholera cases occurring in the population may increase. Further surveillance and research is needed for outbreak control, risk assessment and prevention of O139 cholera.

\section{ACKNOWLEDGEMENTS}

This work was funded by the Mega-projects of Science and Technology Research of China (2012ZX10004213), the NSFC of China (30872260, 31570134) and the Guangdong Province Science and Technology Plan Projects (no. 2014A020219004).

\section{DECLARATION OF INTEREST}

None.

\section{REFERENCES}

1. Albert MJ, et al. Large outbreak of clinical cholera due to Vibrio cholerae non-O1 in Bangladesh. Lancet 1993; 341: 704.

2. Ramamurthy $\mathbf{T}$, et al. Emergence of novel strain of Vibrio cholerae with epidemic potential in southern and eastern India. Lancet 1993; 341: 703-704.

3. Comstock LE, et al. Cloning and sequence of a region encoding a surface polysaccharide of Vibrio cholerae O139 and characterization of the insertion site in the chromosome of Vibrio cholerae O1. Molecular Microbiology 1996; 19: 815-826.

4. Li M, et al. Comparative genomic analyses of the vibrio pathogenicity island and cholera toxin prophage regions in nonepidemic serogroup strains of Vibrio cholerae. Applied and Environmental Microbiology 2003; 69: 1728-1738.

5. Olsvik O, et al. Use of automated sequencing of polymerase chain reaction-generated amplicons to identify three types of cholera toxin subunit B in Vibrio cholerae O1 strains. Journal of Clinical Microbiology 1993; 31: 22-25.

6. Nair GB, et al. New variants of Vibrio cholerae O1 biotype El Tor with attributes of the classical biotype from hospitalized patients with acute diarrhea in Bangladesh. Journal of Clinical Microbiology 2002; 40: 3296-3299.

7. Bhuiyan NA, et al. Changing genotypes of cholera toxin (CT) of Vibrio cholerae O139 in Bangladesh and description of three new CT genotypes. FEMS Immunology and Medical Microbiology 2009; 57: 136-141.

8. Zhang P, et al. Novel ct $x B$ variants of Vibrio cholerae O1 isolates, China. Infection Genetics and Evolution 2013; 20: 48-53.

9. Zhang $\mathbf{P}$, et al. The 7 th pandemic Vibrio cholerae $\mathrm{O} 1 \mathrm{El}$ Tor isolate in China has undergone genetic shifts. Journal of Clinical Microbiology 2014; 52: 964-967.

10. Liu J, et al. Diarrhea outbreak by Vibrio cholerae O139 in Keping Xinjiang [in Chinese]. Disease Surveillance 1993; 8: 238-239.

11. Zhang P, et al. A molecular surveillance reveals prevalence of Vibrio cholerae $\mathrm{O} 139$ isolates in China, 1993-2012. Journal of Clinical Microbiology 2014; 52: 1146-1152.

12. World Health Organization. Manual for Laboratory Investigations of Acute Enteric Infections. Edited by the Diarrhoeal Disease Control Programme. Geneva: World Health Organization, 1987, pp. 1-113.

13. Shi L, et al. Detection of genes encoding cholera toxin (cholera toxin), zonula occludens toxin (ZOT), accessory cholera enterotoxin (ACE) and heat stable enterotoxin (ST) in Vibrio mimicus clinical strains. Microbiology and Immunology 1998; 42: 823-828.

14. Rivera IN, et al. Genotypes associated with virulence in environmental isolates of Vibrio cholerae. Applied and Environmental Microbiology 2001; 67: 2421-2429.

15. Singh DV, et al. Molecular analysis of Vibrio cholerae O1, O139, non-O1, and non-O139 strains: clonal relationships between clinical and environmental isolates. Applied and Environmental Microbiology 2001; 67: 910-921. 
16. Chow KH, et al. Detection of RTX toxin gene in Vibrio cholerae by PCR. Journal of Clinical Microbiology 2001; 39: 2594-2597.

17. Centers for Disease Control and Prevention. Rapid standardized laboratory protocol for molecular subtyping of Vibrio cholerae by pulsed field gel electrophoresis (PFGE), 2006.

18. Son MS, et al. Characterization of Vibrio cholerae O1 El Tor biotype variant clinical isolates from Bangladesh and Haiti, including a molecular genetic analysis of virulence genes. Journal of Clinical Microbiology 2011; 49: 3739-3749.

19. Raychoudhuri A, et al. Classical ctxB in Vibrio cholerae O1, Kolkata, India. Emerging Infectious Diseases 2009; 15: $131-132$.
20. Tran HD, et al. Multi-drug resistant Vibrio cholerae O1 variant El Tor isolated in northern Vietnam between 2007 and 2010. Journal of Medical Microbiology 2012; 61: 431-437.

21. Tang X, et al. Outbreak of cholera associated with consumption of soft-shelled turtles, Sichuan province, China, 2009 [in Chinese]. Chinese Journal of Epidemiology 2010; 31: 1050-1052.

22. Li H, et al. Analysis of the source of a cholera outbreak caused by 0139 Vibrio cholerae [in Chinese]. Disease Surveillance 2008; 23: 218-220.

23. Qiu X, et al. Investigation on a foodborne cholera O139 outbreak in a rural banquet and the turtle markets [in Chinese]. Practical Preventive Medicine 2013; 20: 171-174. 